\title{
DDRD Signature
}

National Cancer Institute

\section{Source}

National Cancer Institute. DDRD Signature. NCI Thesaurus. Code C129891.

A gene expression profile that indicates that the cells in a sample have decreased ability to respond to and repair DNA damage. 